\title{
14. COMMISSION DES DONNEES SPECTROSCOPIQUES FONDAMENTALES
}

\author{
Report of Meetings, 23 and 25 August 1967
}

President: Charlotte Moore-Sitterly.

SeCretary: J.G. Phillips.

Silent tribute was paid to the memory of three members lost by death since the 1964 meeting: J.E. Mack, W.F. Meggers, G. Racah; and of one consultant, G.H. Dieke.

Corrections to the Draft Report were submitted in writing to the President. They were not extensive.

Unfortunately, no offprints of the Report will be available for general distribution, but the President hopes to have a number of copies made and distributed to interested workers.

It was decided that the present subcommittees $2 \mathrm{a}$ and $2 \mathrm{~b}$ be combined to form one committee on 'Atomic Collision Cross Sections and Line Broadening'. An additional committee on 'Structure of Atomic Spectra' was, also, recommended.

R.W. Nicholls, Director, Centre for Research in Experimental Space Science, York University, Toronto, Canada, has been given the support of Commission 14 as a sponsor for an International Symposium on Laboratory Astrophysics, to be held at York University in 1969.

A.G.W. Cameron brought to the attention of Commission 14 the question of the possible formation of a Joint Commission on Extraterrestrial Chemistry.

CODATA held its second annual meeting in Moscow on May 31, June 1, 2, 1967. It was suggested that smaller countries be given membership in CODATA and on National Committees, with the Academy of the country as a link. The Executive Staff has begun to assemble a directory containing information on continuing data projects all over the world.

Relevant to this, a report by Nils Aslund has been submitted to the President 'On the Problem of Sorting and Presenting Results of Molecular Spectroscopic Studies'. The problem is one of communicating numerical information from one experimentalist to another or from experimentalists to theoreticians. The idea of an information bank from which information could be distributed, was discussed at a seminar on molecular physics held in Blaricum, Holland, March 29-31 under the leadership of C.M. Moser. This report has been submitted by the President to G. Waddington to be considered by CODATA.

The Secretary General of IUPAC, R. Morf, submitted a list of suggestions on Nomenclature, Symbols and Usage in Analytical Atomic Spectroscopy. It was pointed out that this general question should be handled by the newly-formed Triple Commission for Spectroscopy.

The President made the following interim suggestions regarding the list:

The use of small caps to denote a spectrum, i.e. $\mathrm{Mg}$ I rather than $\mathrm{Mg} \mathrm{I}$.

The use of $E P$ rather than $V_{\mathrm{e}}$ for excitation potential.

The use of $I P$ rather than $V_{1}$ for ionization potential.

\section{Scientific Meeting 23 August, 1967}

B. Edlen, Chairman of the Committee on Standards of Wavelength, proposed for discussion the following topics:

(1) Stabilization of laser wavelengths.

(2) The primary standard:

(a) The wavelength of the $\mathrm{Kr}^{86}$ line in different light sources

(b) Origin of the observed asymmetry in the profile of the $\mathrm{Kr}^{86}$ line 
(3) Rare-gas standards.

(4) Wavelength standards in the vacuum ultraviolet.

(5) The refractive index of air.

With regard to topic 5 , reference was made to a paper by J.C. Owens (6) confirming the validity of the Edlén equation (1) within the usual ranges of temperature and pressure.

The discussion in the Draft Report concerning vacuum-ultraviolet standards was briefly reviewed.

The question of the actual wavelength of the $\mathrm{Kr}^{86}$ primary standard line in light sources other than the Engelhard hot-cathode lamp (topic 2a) had arisen in connection with Norlén's measurements referred to in the Draft Report in which he produced the primary standard by means of a microwave-excited electrodeless lamp obtained from Dr. Engelhard.

The suggestion that the wavelength of the primary standard line emitted by this electrodeless lamp should be calibrated against that of the adopted standard lamp met with a gratifying response from members of the committee.

Terrien pointed out that the primary standard is defined in terms of conditions which are impossible to attain in practice. However, it has been shown that there is a $1: 10^{8}$ agreement between theory and practical lamps. The same meter steel gauge measured at different laboratories using different sources have agreed within $10^{8}$. The electrodeless lamp mentioned by Edlén is not a standard, so one does not know the actual wavelength, but it could be very easily checked against a standard lamp. Terrien reported that agreement between $\mathrm{Hg}^{198}$ lamps was $0.0012 \AA$.

Cook asked what was the width of the line in the electrodeless Engelhard lamp. Edlén replied that the profile had not been measured. Cook commented that the broadening is probably mainly Doppler; that he could undertake to make wavelength and width checks. Cook agreed with Terrien regarding the measurement of the meter, finding a few parts in $10^{8}$ agreement.

Baird reported finding a reproducibility of 1 to $2: 10^{9}$ in the wavelength of lamps producing the standard source, and he offered to make wavelength measurements for Edlén at NRC.

To the question of the observed asymmetry of the primary standard line and other $\mathrm{Kr}^{\mathbf{8}}$ lines Engelhard reported that the purity of the $\mathrm{Kr}^{86}$ samples was better than $99.5 \%$ as measured by mass spectroscopy. This would appear to exclude the possibility that the asymmetry could be caused by a satellite due to one of the lighter isotopes. Cook confirmed that the observed profiles of the standard line could hardly be explained in terms of isotopic contamination. The cause of the asymmetry remains unknown.

Cook reported that solar spectroscopy requires the establishment of several standard line profiles of $\mathrm{Kr}^{86}$ and $\mathrm{Hg}^{198}$. Of value are the yellow and violet lines of $\mathrm{Hg}^{198}$ and its green line, although the temperature must be controlled very carefully.

Terrien reported the publication (7) of profiles of lines of $\mathrm{Kr}^{86}$. Baird called attention to an agreement with the Australian workers that the a symmetry of the $\mathrm{Kr}^{86}$ standard line is $0.02 \mathrm{~m}^{-1}$.

The chairman called on Dr. Engelhard to report on the stability of la ser wavelengths. Engelhard stated that they have had considerable experience at PTB using the He-Ne laser in metrology and gave a detailed presentation of their studies concerning the dependence of the laser wavelength on various experimental conditions. With all variables taken into account the wavelength of the $\mathrm{He}-\mathrm{Ne}^{20}$ laser had been found to range from 6329.9136 to $6329.9146 \AA$. This accuracy is sufficient for practical measuring purposes, and for such purposes the $\mathrm{He}-\mathrm{Ne}^{20}$ laser is considered as an excellent working standard.

Baird plans to correlate emission from lasers with the primary standard as a control when using them in metrology. He wonders whether one should ever consider a laser source as more than a poor secondary standard. He is planning to use high power tunable lasers and will seek to find a ground state line in the visible. Such a line, he believes, will have the most freedom from the troubles indicated above.

Cook agreed with the comments of Baird. He asked whether a rare gas might not be used to make a stable maser. He intends to try to stabilize a molecular maser. He emphasized, (1) that the width of the line was important in the He-Ne laser, and (2) that the electronic properties of the servo synchronization system are very important for the stability of the wavelengths to be measured. 
Terrien agreed with both Baird and Cook. He plans to use the He-Ne laser only when the wavelength is not important. However, he thinks that eventually standard lines will be found. For purpose of metrology, it would be an advantage to find a longer wavelength line.

Kessler reported that at NBS they are using absorption techniques for stabilizing laser wavelengths.

\section{Second Meeting, 23 August 1967}

C.J. Humphreys reported on improved interferometric wavelength measurements of $\mathrm{Xe}^{136}$ and $\mathrm{Kr}^{86}$.

The pair-coupling detected in the $f-g$ transitions of the rare gases in the $4 \mu$ region is described in detail in a recently published paper (3).

To the above report by Humphreys on rare-gas standards the chairman commented that G. Norlén at Lund had recently measured many of the same $\mathrm{Kr}^{86}$ lines (5). The agreement with the values now reported by Humphreys is very good except for a small systematic difference in part of the region.

\section{Transition Probabilities}

R.H. Garstang, Chairman of the Committee on Transition Probabilities, reviewed recent work on this subject and on cross sections. He drew attention to experimental work on forbidden transitions in $\mathrm{Pb} \mathrm{I}$ and $\mathrm{Pb}$ II (2) and I I (4) which materially increase our confidence in the correctness of the transition probabilities of forbidden lines. For allowed transitions attempts to include configuration interaction in the theory are very important.

The President closed the session with some remarks on Future Trends in Spectroscopy.

\section{Standards of Wavelength}

Sustained effort must be made to extend the work on standards to shorter wavelengths, since optical stellar spectra now overlap the soft $\mathrm{X}$-ray region. It is, also, conceivable that standards of wavelength for X-ray spectra may well come under the domain of this Commission, particularly in the range where optical and soft X-ray spectra overlap. The work of Commission 44 points to future needs that must be recognized.

At the time when plans were being made to initiate the large program on 'Atomic Energy Levels' at the National Bureau of Standards, there was a request that the program include X-ray, as well as, optical spectra. This is already becoming a problem of astrophysical interest.

\section{Transition Probabilities}

The demand for experimental data of high quality is so great that work in this field will continue to expand.

\section{Solar and Other Stellar Spectra}

Present solar spectra extend from near $6 \AA$ to well beyond $25000 \AA$. In the 'accessible' and near infrared region the new solar atlases in course of preparation at Liège, based on observations at the Jungfraujoch, as well as those from Michigan and elsewhere, all indicate the presence of many more faint solar lines than are at present recorded. At least $25 \%$ of the known solar lines over this range are still unidentified. It is hoped that the real solar spectrum in the near infrared can be observed above the earth's atmosphere. Most of this region is still unexplored.

To shorter waves Tousey and his associates report more than 7000 solar lines between $2000 \AA$ and $3000 \AA$, of which only about $52 \%$ are identified. Other stellar spectra will, also, require interpretation in the ultraviolet as observing techniques improve. Ultraviolet stellar spectroscopy is still in its infancy.

Many lines to longer waves in high dispersion stellar spectra still defy identification. It is shocking 
to realize that at least 88 atomic lines in the sun spot spectrum remain unidentified, to say nothing of the thousands of molecular spot lines that have never been measured. Here is a serious challenge to the spectroscopists, both atomic and molecular.

\section{Laboratory Spectra}

A sudden revival of interest in highly-ionized atomic spectra has resulted from the observations of the ultraviolet solar spectrum. A wide gap exists between the demand and the supply for analyses of spectra of abundant elements. An illustration will serve to describe the general picture. The total number of silicon spectra to be expected is 14 . Lines from each of the 14 spectra are present in the solar spectrum. The span of wavelengths covered is $6 \AA$ to $25129 \AA$, and the range of ionization potentials is 8 to $2673 \mathrm{eV}$.

In the Draft Report, selected spectra of a number of elements are mentioned, which are being studied at Lund, Culham, and other laboratories. The present remarks are limited mostly to those spectra which would well repay further study, but are not on scheduled programs. As is the case with $\mathrm{Si}$, more emphasis should be given to the spectra of intermediate stages of ionization (IV and greater) from $\mathrm{K}$ thru $\mathrm{Ni}$.

Some third spectra have been seriously neglected, notably the spectra of the inert gas type, but this is now being recognized. $\mathrm{Cr}$ III is scheduled, but it is still in an unfinished state so far as analysis is concerned. It may well be of interest to stellar workers. The spectra of phosphorus above the second stage of ionization should be completely reobserved and analyzed.

A number of "well-known" spectra should be reobserved with modern sources and equipment. Analyses could be extended for Sc I and Sc II. Notable additions could be made to the first and second spectra of the familiar elements $\mathrm{Fe}, \mathrm{Co}, \mathrm{Ni}$. Of these, $\mathrm{Fe}$ Il is being investigated by L.C. Green; and $\mathrm{Ni}$ II has been reobserved by A. G. Shenstone, who is finding hundreds of new lines that as yet have defied interpretation. Monographs are needed also, on $\mathrm{Sr}$ I and $\mathrm{Ba}$ I.

As revisions of individual spectra are completed, the writer hopes to include the data in the new series of publications which will contain revised Tables of 'Atomic Energy Levels' and of 'Multiplets'.

\section{Rare Earth Spectra}

These spectra provide both an interesting and a difficult challenge. At long last $\mathrm{Ce} \mathrm{I}$ is being analyzed. For years this very complex spectrum defied analysis in the hands of talented spectroscopists. Now $\mathrm{Ce}_{\mathrm{I}}$, as observed with improved sources, is known to have some 22000 lines of which about 17000 have been classified, according to W.C. Martin. The progress with other lanthanon spectra is summarized on pp. 253-254 of the Draft Report.

Special mention should be made of $\mathrm{Yb}$ II. The late W.F. Meggers worked on this spectrum for over 30 years and collaborated with the late $\mathrm{G}$. Racah for more than 15 years on the interpretation. The final Monograph has just gone to press. Unfortunately, Meggers did not live to see this 'magnum opus' thru publication. It was his desire that $\mathrm{Yb}$ II be the model to inspire and guide others in their studies of rare earth spectra. Meggers' painstaking care in observing and interpreting this complex spectrum and the Zeeman patterns has resulted in the classification of more than 4000 lines, in the range $2018 \AA$ to $24377 \AA$, from 141 even levels and 174 odd levels.

Enough illustrations have been given to indicate that with suitable modern light sources such as the electrodeless lamp, sliding spark, etc. and good equipment, improved line lists of correctly separated spectra of atoms and ions can be obtained. Many spectra formerly classed as 'well-known' can be studied further to great advantage.

\section{Summary}

Many other topics might be mentioned: autoionization, forbidden lines, laser research, etc. Critically compiled spectroscopic data will be in greater demand as time goes on, and international cooperation will become more essential as the demand increases. It is the responsibility of Commis- 
sion 14 to be alert to the needs of the astrophysicist and to collaborate to the utmost extent in fulfilling these needs. Not only is this a responsibility; it is a rewarding duty in the interesting era ahead.

Chairman: M. Migeotte.

\section{Third Meeting, 25 August 1967}

The last two scientific sessions dealt with molecular spectra. Recent work on diatomic molecules at the National Research Council of Canada was reported by G. Herzberg. New bands of $\mathrm{B}_{2}, \mathbf{B H}$, $\mathrm{Si}_{2}$ and $\mathrm{SiH}$ were observed by the method of flash photolysis. In $\mathrm{CH}$ a Rydberg Series was observed, giving the ionization potential $10 \cdot 64 \mathrm{eV}$.

Absorption spectra of molecular ions were observed by means of flash discharges. Two new states of $\mathrm{C}_{2}$ were found but they are not easy to explain. $\mathrm{SiC}_{2}$ has also been observed at low temperature in absorption at Ottawa.

G. Herbig gave a general talk on 'Interstellar Molecules' in which he described possible processes of formation of diatomic molecules in space; suggesting that two-body radiative recombination may be operating. From observations of $\mathrm{CH}$ (the 0.0 band at 3143) an $f$-value $=3 \times 10^{-3}$ has been derived.

A search for accessible triatomic molecules in the spectrum of $\zeta$ Oph has been unfruitful.

He stressed the need for more laboratory observations and analyses, particularly of the ground states of the hydrides; for absolute $f$-values (of $\mathrm{CH}^{+}$, for example); and for photoionization and photodissociation cross sections.

Chairman: G. Herzberg.

\section{Fourth Meeting, 25 August 1967}

In the last session, A. Lagerqvist reported on recent laboratory work at Stockholm on 'Diatomic Spectra'. Many diatomic molecules of hydrides and oxides have been observed and analyzed. The papers from this laboratory will be in more and more demand by astrophysicists. $P$. Swings made a special request that $\mathrm{FeH}$ be put on the program at Stockholm.

The final talk was by J.G. Phillips, Chairman of the Committee on Molecular Spectra, who described the Berkeley Program. The first Monograph of the Berkeley Series, CN, was published in 1963. In the second Monograph of this series, 35 bands of $C_{2}$ are tabulated and analyzed. Successive publications will give similar data on $\mathrm{TiO}$ and $\mathrm{ZrO}$.

The program is designed to help the astrophysicist. For example, the $\mathrm{C}_{2}$ analysis is being used to study some sixteen carbon stars. The low ratio of $\mathrm{C}^{12} / \mathrm{C}^{13}, 2$ to 1 , in $\mathrm{Y}$ Can Ven is not permitted by Fowler's present theory. Much more study of this problem is needed. The $\mathrm{C}_{2}$ work is being used, also, to revise the identifications in the new solar Monograph. Other illustrations could be given, but these serve to show the present need for such programs.

REFERENCES

1. Edlén, B. 1966, Metrologia, 2, 71.

2. Hults M.E. 1966, J. opt. Soc. Am., 56, 1298.

3. Humphreys, C.J., Paul Jr., E., Cowan, R.D., Andrew, K.L. $\quad$ 1967, J. opt. Soc. Am., $57,855$.

4. Husain, D., Wiesenfeld, J.R. 1966, Nature, 213, 1227.

5. Norlén, G. 1967, Ark. Fys., in press.

6. Owens, J.C. 1967, Appl. Opt., 6, 51.

7. Terrien, J. $\quad$ 1967, J. Phys. (Paris) 28, suppl. p. C 2-3. 